\title{
Partial Kondo screening in frustrated Kondo lattice systems
}

\author{
Yukitoshi Motome, Kyoya Nakamikawa, Youhei Yamaji, and Masafumi Udagawa \\ Department of Applied Physics, University of Tokyo, Hongo, Tokyo 113-8656, Japan
}

(Dated: June 24, 2018)

\begin{abstract}
We investigate the effect of geometrical frustration on the competition between the Kondo coupling and the Ruderman-Kittel-Kasuya-Yosida interaction in Kondo lattice systems. By variational Monte Carlo simulations, we reveal an emergent quantum phase with partial ordering in which the frustration is relieved by forming a magnetic order on a sublattice and leaving the rest in the Kondo screening with spin-singlet formation. The role of quantum fluctuations and spin-charge interplay is elucidated.

PACS numbers: 71.27.+a, 75.30.Mb, 75.20.Hr
\end{abstract}

Kondo lattice systems provide a fertile ground for studying fascinating phenomena in strongly-correlated electron systems [1]. The key concept is competition between the Kondo coupling and the Ruderman-KittelKasuya-Yosida (RKKY) interaction. The former is a local antiferromagnetic (AFM) interaction between conduction electrons and localized moments, which promotes spin-singlet formation and results in Fermi liquid states, as the so-called Kondo effect 22. On the other hand, the latter RKKY interaction is an effective magnetic coupling between localized moments mediated by conduction electrons, which tends to stabilize a magnetic ordering [3]. The competition leads to a quantum critical point (QCP) between a Fermi liquid state and a magnetically ordered state [4]. QCP has attracted much attention as a source of fascinating phenomena, such as non-Fermi-liquid behavior and superconductivity.

In the present study, we explore yet another phenomenon emergent from the competition between the Kondo coupling and the RKKY interaction. Our interest is in the possibility to have an intermediate quantum phase induced by geometrical frustration, which preempts QCP, with a coexistence of screened local moments due to the Kondo singlet formation and magnetically ordered moments stabilized by the RKKY interaction. This is a partially ordered state, which we call the partial Kondo screening (PKS) state in this paper.

The partial ordering is sometimes seen in localized spin systems with geometrical frustration [5]. Our target PKS is, however, qualitatively different from the partial ordering in localized spin systems in the following points: The system includes itinerant electrons, and the disordered sites are not simply paramagnetic but participate in the Kondo singlet formation with vanishing their moments. These differences will bring about many distinctive aspects not only in the stabilization mechanism of the partial order but also in the resulting physical properties.

Several candidates for PKS are experimentally found in $f$-electron compounds, e.g., a distorted kagome material $\mathrm{CePdAl}\left[\underline{6}\right.$, ,7] and a triangular material $\mathrm{UNi}_{4} \mathrm{~B}[\mathbf{8}, 9]$. These PKS states were theoretically studied by the meanfield approximation of a pseudomoment model, which describes the magnetic and singlet states by discrete classical variables [10 12. In the previous studies, the effects of quantum fluctuations and the interplay between conduction electrons and localized moments are not fully taken into account, despite the fact that they are obviously crucial in the spin-charge coupled systems.

In this Letter, we explore PKS as a quantum phase for the Kondo lattice model and the Kondo necklace model on frustrated lattices. The Kondo lattice model (KLM) is one of the fundamental models for rare-earth compounds, whose Hamiltonian reads

$$
\mathcal{H}=-t \sum_{\langle i j\rangle \sigma}\left(c_{i \sigma}^{\dagger} c_{j \sigma}+\text { h.c. }\right)+J \sum_{i} \boldsymbol{\tau}_{i} \cdot \boldsymbol{S}_{i}+I_{z} \sum_{\langle i j\rangle} S_{i}^{z} S_{j}^{z}
$$

where the first term describes the hopping of conduction electrons and the second term represents the Kondo coupling between the conduction electron spin $\boldsymbol{\tau}_{i}$ and the localized spin $\boldsymbol{S}_{i}$. For simplicity, we consider $S=1 / 2$ spins for the localized spins. In Eq. (1) we extend the model by adding the last term, the AFM Ising interaction between localized spins $\left(S_{i}^{z}\right.$ is the $z$ component of $\boldsymbol{S}_{i}$ ), in order to mimic the magnetic anisotropy often seen in real materials 13. The Kondo necklace model (KNM) is a simplified variant of KLM at half filling [4]:

$$
\mathcal{H}=W \sum_{\langle i j\rangle} \boldsymbol{\tau}_{i} \cdot \boldsymbol{\tau}_{j}+J \sum_{i} \boldsymbol{\tau}_{i} \cdot \boldsymbol{S}_{i}+I_{z} \sum_{\langle i j\rangle} S_{i}^{z} S_{j}^{z},
$$

where the charge degree of freedom of conduction electrons is suppressed with assuming that there is one electron localized at every site. Instability toward PKS in KNM was studied previously by the authors for limited lattice geometries and system sizes [14]. We consider the two models on one of the simplest frustrated lattices, the two-dimensional triangular lattice, and take the sums $\langle i j\rangle$ over the nearest-neighbor sites.

We study the ground state of the models (11) and (2) by the variational Monte Carlo (VMC) method. The method has several advantages compared to others; e.g., it takes account of quantum fluctuations neglected in the mean-field approximation, and it can avoid the minus sign problem even in frustrated systems. We here consider the variational wave function in the form

$$
|\psi\rangle=P_{\mathrm{G}} \mathcal{L}^{S=0} \mathcal{L}^{K=0}\left|\phi_{\text {pair }}\right\rangle,
$$


which describes magnetic states and nonmagnetic singlet states on an equal footing as a natural extension of the Yosida-type wave function [15]. Here $\left|\phi_{\text {pair }}\right\rangle$ is a generalized BCS wave function defined by $\left|\phi_{\text {pair }}\right\rangle=$ $\left(\sum_{\ell, m=1}^{2 N} f_{\ell m} a_{\ell \uparrow}^{\dagger} a_{m \downarrow}^{\dagger}\right)^{N_{\mathrm{e}}}|0\rangle$, where $f_{\ell m}$ are the variational parameters and $|0\rangle$ is a vacuum: The fermion operators $a_{\ell \sigma}$ represent both conduction $c_{i \sigma}$ and localized electrons (or localized spins), resulting in pair creations for any combination of them. We focus on the half-filling case by setting $N_{\mathrm{e}}=N$, where $N$ is the number of lattice sites. The electron number is fixed to be one at each localized spin site. $\mathcal{L}^{S=0}$ and $\mathcal{L}^{K=0}$ are the quantum-number projection operators for the total spin singlet and the total momentum zero, respectively. $P_{\mathrm{G}}$ is the Gutzwiller factor for optimizing the weight of configurations with double occupancies in KLM; $P_{\mathrm{G}}=\exp \left(-\sum_{i} g_{i} n_{i \uparrow} n_{i \downarrow}\right)$ where $g_{i}$ are the variational parameters and $n_{i \sigma}=c_{i \sigma}^{\dagger} c_{i \sigma}$. We follow Ref. [16] to optimize a large number of variational parameters by using the stochastic reconfiguration [17] and to enforce the quantum-number projections.

In the present study, we explore the solutions with three-sublattice ordering by imposing $\mathcal{L}^{K=0}$ only for the same sublattices and by considering only the sublattice dependence of $g_{i}$ in $P_{\mathrm{G}} . \quad \mathcal{L}^{S=0}$ is used only for $I_{z}=$ 0 . Typically, the optimization is achieved by $300-1000$ stochastic reconfiguration steps with 1600-6000 Monte Carlo samplings. We confirm that the optimized wave function gives a precise ground-state energy compared to the results by the exact diagonalization; e.g., the relative error is less than 0.03 for KNM with $N=12$. We apply the method to the clusters with system size $N=12,18$, and 24 with imposing the boundary conditions compatible with the three-sublattice order.

First we discuss the results for KNM given by Eq. (2). Figure 1(a) summarizes the ground-state phase diagram determined by VMC. There are three distinct regions, i.e., a magnetically-ordered (MO) state in the small $J / W$ region, a Kondo spin liquid (KSL) state in the large $J / W$ region, and a PKS state in between. The MO state for small $J / W$ has a three-sublattice ordering as schematically shown in the figure. This peculiar order is governed by the first and third terms in the Hamiltonian (21) as discussed later. In the opposite large- $J / W$ region, the second term becomes dominant, and the Kondo singlet is formed at every site to realize KSL. In the intermediate competing regime, we obtain the PKS phase in which one sublattice is dominated by the local Kondo singlet formation and the remaining two retain magnetic ordering, as schematically depicted in the figure.

Then we show how we identify three phases in the following. Figure 2 plots $J / W$ dependences of the onsite correlation $\left\langle\boldsymbol{\tau}_{i} \cdot \boldsymbol{S}_{i}\right\rangle_{\mu}$ and the intersite correlation between the localized spins on the same sublattice $\left\langle\boldsymbol{S}_{i} \cdot \boldsymbol{S}_{j}\right\rangle_{\mu}(\mu$ denotes $\mathrm{A}, \mathrm{B}$, or $\mathrm{C})$. In the intermediate $J / W$ region, the onsite correlation $\left\langle\boldsymbol{\tau}_{i} \cdot \boldsymbol{S}_{i}\right\rangle_{\mu}$ on one sublattice (C in this case) becomes considerably larger in magnitude than the other two, as shown in Figs. 22(a)-(c). The difference $\Delta=\left|\left\langle\boldsymbol{\tau}_{i} \cdot \boldsymbol{S}_{i}\right\rangle_{\mathrm{C}}-\left(\left\langle\boldsymbol{\tau}_{i} \cdot \boldsymbol{S}_{i}\right\rangle_{\mathrm{A}}+\left\langle\boldsymbol{\tau}_{i} \cdot \boldsymbol{S}_{i}\right\rangle_{\mathrm{B}}\right) / 2\right|$ is plotted
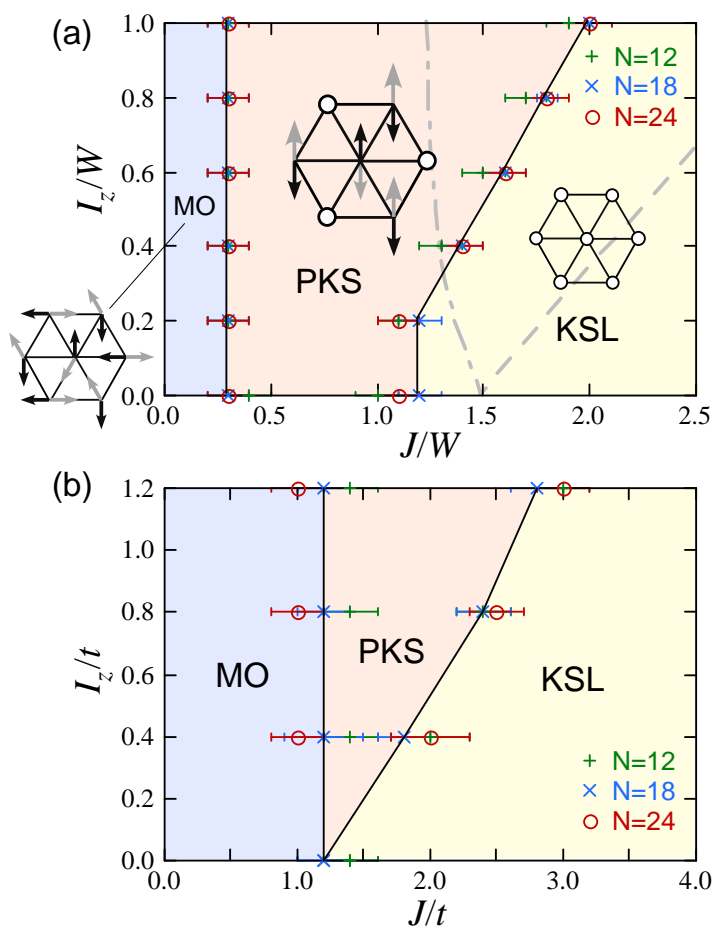

FIG. 1: (color online). Phase diagrams for (a) the Kondo necklace model and (b) the Kondo lattice model at half filling on the triangular lattice determined by VMC. The lines are guides for the eye connecting the data for $N=18$. There are three phases: MO, PKS, and KSL. Schematic pictures for the spin state in each phase are shown in (a), where the gray (black) arrows represent $\boldsymbol{\tau}_{i}\left(\boldsymbol{S}_{i}\right)$ and the circles denote the Kondo singlets. The dot-dashed and dashed lines in (a) show the phase boundaries for MO-PKS and PKS-KSL, respectively, determined by the mean-field approximation.

for different system sizes $N$ in each inset. In the same region, $\left\langle\boldsymbol{S}_{i} \cdot \boldsymbol{S}_{j}\right\rangle_{\mathrm{C}}$ is suppressed compared to those for A and $\mathrm{B}$ sublattices, and moreover, it decays quicker with increasing distance than the other two [Figs. 2(d)-(f)]. These are clear indications of PKS; the local Kondo singlet is enhanced on the $\mathrm{C}$ sublattice compared to the rest magnetically active sublattices [18].

Such specific spin configuration in the PKS state is also evidenced by the intersite correlations among different sublattices plotted in Figs. 3(a) and 3(b). For both $\boldsymbol{\tau}_{i}$ and $\boldsymbol{S}_{i}$, the intersite correlations measured from the $\mathrm{C}$ sublattice are suppressed, while those between A and B sublattices are robustly AFM. Therefore, the spin configuration of the PKS state is basically composed of enhanced Kondo singlets on one sublattice and the AFM network on the remaining unfrustrated honeycomb lattice, as schematically shown in Fig. 1(a).

In the smaller $J / W$ region, Figs. 3(c) and 3(d) show that $\left\langle\boldsymbol{\tau}_{i} \cdot \boldsymbol{\tau}_{j}\right\rangle$ is almost independent of the sublattice, while $\left\langle\boldsymbol{S}_{i} \cdot \boldsymbol{S}_{j}\right\rangle \simeq \pm 1 / 4$ between A and B but $\left\langle\boldsymbol{S}_{i} \cdot \boldsymbol{S}_{j}\right\rangle \simeq 0$ between $\mathrm{C}$ and other sublattices. These behaviors indicate the three-sublattice order for the MO phase in Fig. I(a): 

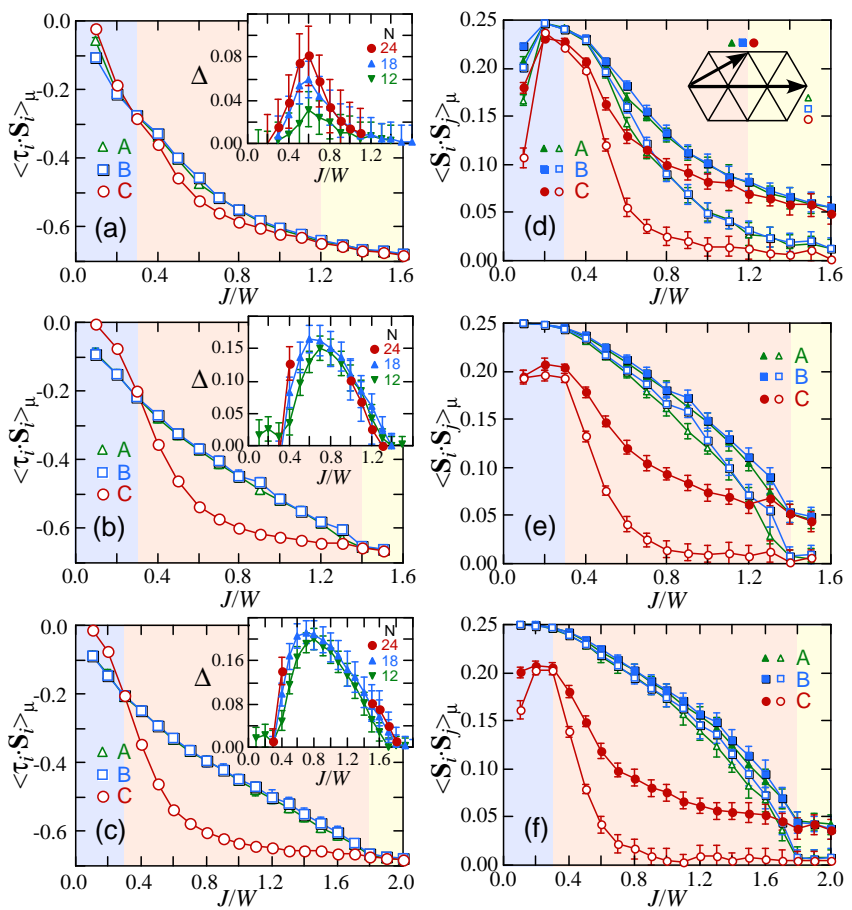

FIG. 2: (color online). (a)-(c) Onsite correlation for the threesublattice $\mu=\mathrm{A}, \mathrm{B}, \mathrm{C}$. The insets show their difference $\Delta$ for various system sizes $N$. (d)-(f) Intersite correlation between localized spins on the same sublattice. Closed and open symbols show the results for the distance $\sqrt{3}$ and 3 , respectively, as shown in the inset in (d). Data in the main panels are for KNM with $N=18$ and at (a),(d) $I_{z}=0.0$, (b),(d) $I_{z} / W=0.4$, and (c),(f) $I_{z} / W=0.8$. The lines are guides for the eye.

$\boldsymbol{\tau}_{i}$ forms almost $120^{\circ}$ order to optimize the $W$ term, while $\boldsymbol{S}_{i}$ exhibits an almost collinear AFM order in A and B sublattices with leaving perpendicular spins on the C sublattice to optimize the $I_{z}$ term.

On the other hand, for large $J / W$, all $\left\langle\boldsymbol{\tau}_{i} \cdot \boldsymbol{S}_{i}\right\rangle_{\mu}$ become identical within the statistical errorbar and take a value close to $-3 / 4$, as shown in Figs. 2(a)-(c). At the same time, all $\left\langle\boldsymbol{S}_{i} \cdot \boldsymbol{S}_{j}\right\rangle_{\mu}$ merge and become small with showing a rapid decay with the distance, as plotted in Figs. 2(d)(f). These are a sign of the KSL phase where the spins form singlets locally as $\left\langle\boldsymbol{\tau}_{i} \cdot \boldsymbol{S}_{i}\right\rangle_{\mu} \sim-3 / 4$.

The phase boundaries are determined from the behaviors of these spin correlations. In particular, the MOPKS boundary is determined by a sudden change of intersite spin correlations in Figs. 2(d)-(f) as well as by the onset of $\Delta$ in Figs. 2(a)-(c). Two states have the same symmetry in terms of spins apparently [18], but we conclude that there is a phase transition between them in the light of the mean-field results discussed below.

Let us make a remark on the limit of $I_{z} \gg J(\gg W)$. In this limit, $\vec{S}_{i}$ are decoupled from $\vec{\tau}_{i}$ and expected to be disordered with macroscopic degeneracy [19]. Since $J$ tends to lift the degeneracy through flipping $\vec{S}_{i}$, the
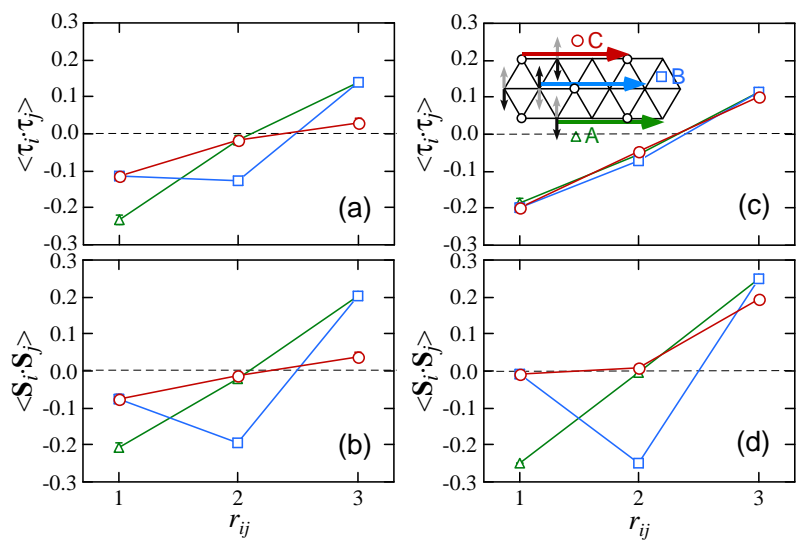

FIG. 3: (color online). Intersite correlation at $I_{z} / W=0.4$ for (a),(b) $J / W=0.6$ and (c),(d) $J / W=0.2$ in the KNM with $N=18$. The correlations are measured along the horizontal arrows in the inset in (c) with fixing the site $i$ at the origins of the arrows and shifting $j$.

system is mapped onto a transverse-field Ising model on the triangular lattice, for which a three-sublattice partial order is suggested to appear [20]. Such consideration leads us to expect the three-sublattice PKS state in the limit of $I_{z} \gg J$; this appears to be consistent with the result in Fig. 1(a).

The VMC phase diagram is compared with that by the mean-field approximation (MFA) in Fig. 1(a). Here we perform MFA which accommodates three-sublattice ordering by extending the method in Ref. [4]. MFA also predicts three phases, MO, PKS, and KSL. However, there are several differences compared with the VMC results. The most distinct one is that VMC predicts the PKS state in a finite range of $J / W$ down to $I_{z}=0$, whereas it disappears at $I_{z}=0$ in MFA. Another important difference is that VMC phase boundaries shift to smaller $J / W$ compared to the MFA results, and the width of MO state becomes relatively narrower. These are consequences of intersite quantum fluctuations neglected in MFA.

Now we turn to KLM in Eq. (10), which includes itinerancy of electrons explicitly. Figure [(b) summarizes the phase diagram at half filling. We find that KLM exhibits a similar sequence of three phases including PKS. The corresponding onsite correlations are plotted in Figs. 4(a)-(c). As seen in KNM [Figs. 22(a)-(c)], $\left\langle\boldsymbol{\tau}_{i} \cdot \boldsymbol{S}_{i}\right\rangle_{\mu}$ becomes larger on one sublattice than the other two in the PKS region. Although $\Delta$ [insets in Fig. 世(b) and 4 (c)] suffers from finite-size effects (the reason is discussed below), we determine phase boundaries from qualitatively similar changes of spin correlations to those in Figs. 2(d)(f) and in Fig. 3 (not shown). At $I_{z}=0$, PKS behavior is not clearly observed in KLM [Fig. 4(a)]. Furthermore, the MO region is wider than in KNM; we return to this point below. Consequently, PKS region becomes relatively narrower compared to the KNM case, yet remains 


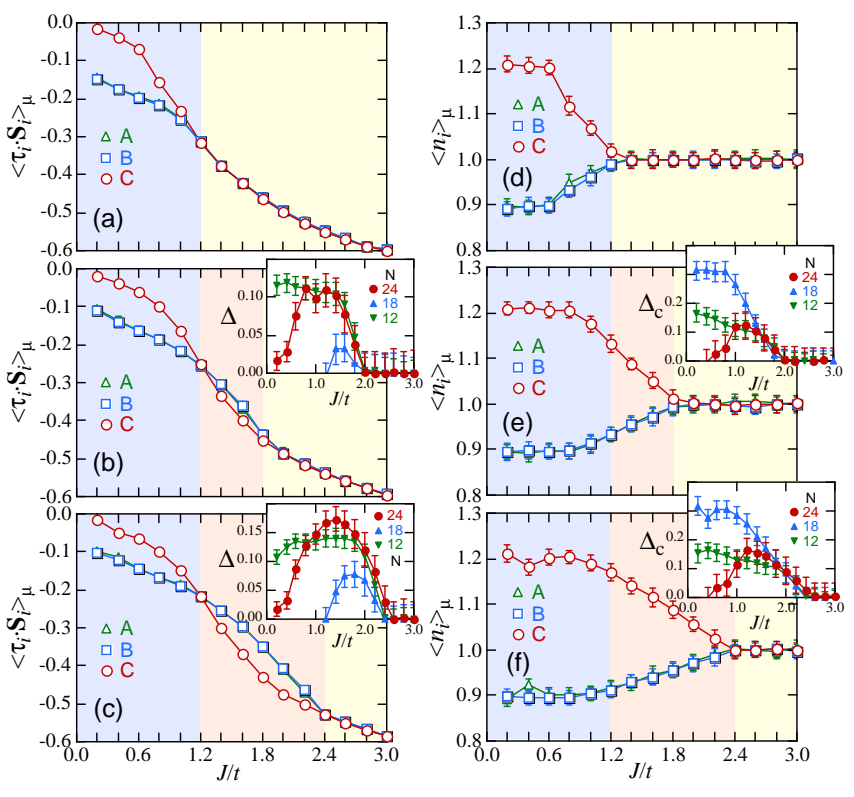

FIG. 4: (color online). (a)-(c) Onsite correlation and (d)-(f) local electron density in KLM with $N=18$. The insets show their differences with varying $N$. The data are for (a),(d) $I_{z}=0.0,(\mathrm{~b}),(\mathrm{d}) I_{z} / t=0.4$, and (c),(f) $I_{z} / t=0.8$.

robust between the MO and KSL phases even when conduction electrons are considered explicitly.

The relatively wider $\mathrm{MO}$ phase is presumably attributed to the complicated role of $J$ in KLM: $J$ enhances the RKKY interaction which tends to stabilize magnetic ordering, in addition to the enhancement of the spinsinglet formation. In addition, the notable system-size dependence in the small $J / t$ region [the insets in Fig. 4] might be due to the long-ranged and oscillating nature of the RKKY interaction [3], which is difficult to capture within the small finite-size clusters. Further study in larger system sizes is desired to clarify the nature of MO in KLM.
Another interesting observation related with the charge degree of freedom in KLM is that PKS accompanies charge disproportionation. The local charge $\left\langle n_{i}\right\rangle_{\mu}=$ $\sum_{\sigma}\left\langle n_{i \sigma}\right\rangle_{\mu}$ disproportionates among the sublattices as shown in Figs. 4(d)-(f). An instability toward charge density wave was recently discussed for the unfrustrated KLM model around quarter filling [21]. The relation is not clear between the instability and our PKS with charge disproportionation. It is interesting to study the possibility of PKS for general filling, in particular, at commensurate filling.

In summary, we have investigated the effect of geometrical frustration on the competition between the RKKY interaction and the Kondo coupling by the variational Monte Carlo simulation for the Kondo lattice model and the Kondo necklace model. The comparative study between two models reveals the following features. (i) Both models exhibit PKS phase in between MO and KSL phases. (ii) The PKS state is further stabilized by quantum fluctuations and the spin anisotropy. (iii) Charge degree of freedom manifests in the stability of the MO phase and in the charge disproportionation associated with PKS. All the results illuminate the appearance of the PKS phase in the Kondo lattice systems even when taking into account quantum fluctuations and spincharge interplay, both of which have not been studied in the previous studies. We believe that the results pave the way for further understanding of PKS observed in complicated materials and of expected spin-charge entangled phenomena inherent to PKS. Our results will also cast a new light on the recent efforts to explore a new paradigm of QCP physics in the Kondo problem [22, 23].

The authors thank D. Tahara for the use of his VMC code and useful comments and T. Misawa for enlightening discussions. This work was supported by KAKENHI (No. 19052008 and No. 21340090), Global COE Program "the Physical Sciences Frontier", and by the Next Generation Super Computing Project, Nanoscience Program, MEXT, Japan.
[1] A. C. Hewson, 'The Kondo Problem to Heavy Fermions' (Cambridge Univ. Press, Cambridge, 1993), and references therein.

[2] J. Kondo, Prog. Theor. Phys. 32, 37 (1964); K. Yosida, Phys. Rev. 147, 223 (1966); P. W. Anderson, J. Phys. C3, 2436 (1970); P. Nozières, J. Low. Phys. 17, 31 (1974).

[3] M. A. Ruderman and C. Kittel, Phys. Rev. 96, 99 (1954); T. Kasuya, Prog. Theor. Phys. 16, 45 (1956); K. Yosida, Phys. Rev. 106, 893 (1957).

[4] S. Doniach, Physica 91B, 231 (1977).

[5] M. Mekata, J. Phys. Soc. Jpn. 42, 76 (1977).

[6] A. Dönni et al., J. Phys.: Cond. Mat. 8, 11213 (1996).

[7] A. Oyamada et al., Phys. Rev. B 77, 064432 (2008).

[8] S. A. M. Mentink et al., Phys. Rev. Lett. 73, 1031 (1994).

[9] A. Oyamada et al., J. Phys.: Cond. Mat. 19, 145246
(2007).

[10] M. D. Núñez-Regueiro, C. Lacroix, and B. Canal, Physica C 282-287, 1885 (1997).

[11] C. Lacroix, B. Canal, and M. D. Núñez-Regueiro, Phys. Rev. Lett. 77, 5126 (1996).

[12] R. Ballou, J. Alloys and Comp. 275-277, 510 (1998), and references therein.

[13] It might be more realistic to incorporate the single-ion anisotropy for $S \geq 1$. To make calculations feasible, we implement the exchange anisotropy for $S=1 / 2$.

[14] Y. Motome, Y. Yamaji, and M. Udagawa, J. Phys.: Conf. Ser. 145, 012068 (2009).

[15] H. Shiba and P. Fazekas, Prog. Theor. Phys. Supplement 101, 403 (1990)

[16] D. Tahara and M. Imada, J. Phys. Soc. Jpn. 77, 114701 (2008), and references therein. 
[17] S. Sorella, Phys. Rev. Lett. 80, 4558 (1998).

[18] PKS state is better identified by the structure factor for $\left\langle\boldsymbol{\tau}_{i} \cdot \boldsymbol{S}_{i}\right\rangle$. This requires the calculation of the four-body correlation function such as $\left(\boldsymbol{\tau}_{i} \cdot \boldsymbol{S}_{i}\right)\left(\boldsymbol{\tau}_{j} \cdot \boldsymbol{S}_{j}\right)$, which is too cpu-time consuming in the present VMC.

[19] G. H. Wannier, Phys. Rev. 79, 357 (1950); R. M. F. Houtappel, Physica 16, 425 (1950); K. Husimi and I. Syôzi, Prog. Theor. Phys. 5, 177 (1950).
[20] R. Moessner and S. L. Sondhi, Phys. Rev. B 63, 224401 (2001).

[21] J. Otsuki, H. Kusunose, and Y. Kuramoto, J. Phys. Soc. Jpn. 78, 034719 (2009).

[22] Q. Si, Physica B 378-380, 23 (2006).

[23] J. Custers et al., Phys. Rev. Lett. 104, 186402 (2010). 